\section{Avaliabilidade do Programa Academia da Saúde no Município do Recife, Pernambuco, Brasil}

\author{
Evaluability of the Health Gym Program in Recife, \\ Pernambuco State, Brazil
}

Disponibilidad del Programa Academia de la Salud en el Municipio de Recife, Pernambuco, Brasil

\section{Resumo}

A implantação do Programa Academia da Saúde é uma das estratégias para a efetivação da Política Nacional de Promoção da Saúde no Brasil, porém, até o momento, não foram realizados estudos de avaliabilidade sobre essa intervenção no Recife, Pernambuco, que se configura como um dos primeiros entes federativos a implantar essa intervenção. Este estudo buscou mensurar a avaliabilidade do Programa Academia da Saúde no Município do Recife, considerando a descrição da intervenção, a elaboração de seus modelos lógicos e teórico de avaliação, além de identificar perguntas avaliativas. Utilizou-se uma abordagem qualitativa com base no sistema de sete elementos, por pesquisa documental, entrevistas e discussão com gestores e técnicos do programa e da técnica da condensação de significados para análise dos documentos e das entrevistas. Verificou-se que o programa apresenta uma extensa gama de objetivos, princípios e diretrizes, e que parte dos gestores desconhece tais elementos. Após a modelagem do programa, observou-se a viabilidade metodológica e operacional para o desenvolvimento de uma pesquisa avaliativa que foi discutida e pactuada com os gestores e trabalhadores.

Avaliação em Saúde; Política de Saúde; Atenção Primária à Saúde, Avaliação de Serviços de Saúde
Rafaela Niels da Silva 1

Flávio Renato Barros da Guarda 1

Pedro Curi Hallal 2

Petrônio José de Lima Martelli 1

doi: 10.1590/0102-311X00159415

\section{Correspondência}

R. N. Silva

Rua Cassilândia 331, Bloco 1, apto. 304, Recife, PE

50740-370, Brasil.

rafanielss@hotmail.com

1 Universidade Federal de Pernambuco, Recife, Brasil.

2 Universidade Federal de Pelotas, Pelotas, Brasil. 


\section{Introdução}

O Ministério da Saúde brasileiro vem implementando desde 2006 uma série de estratégias e políticas setoriais que visam ampliar o escopo das ações na atenção primária à saúde, a prevenção e controle de doenças crônicas e a promoção de estilos de vida saudáveis ${ }^{1}$.

Nesse contexto, destaca-se, em 2011, a instituição do Programa Academia da Saúde, cujo objetivo geral é contribuir para a promoção da saúde pela criação de polos com infraestrutura e recursos humanos qualificados para desenvolver atividades de promoção da saúde e produção do cuidado na atenção primária à saúde 2,3 .

Desde então, a implantação do Programa Academia da Saúde pode se iniciar com a construção de novos polos ou incorporação de programas preexistentes de atividades físicas desenvolvidos nos municípios, que atendam a critérios de similaridade estabelecidos nas portarias normativas do Programa Academia da Saúde 4.

Entre os programas preexistentes que aderiram ao Programa Academia da Saúde, destaca-se o Programa Academia da Cidade, desenvolvido pela Secretaria de Saúde do Recife (Pernambuco) desde 2002 5, que incorporou 37 espaços públicos de lazer às ações do Programa Academia da Saúde, tornando-se um dos primeiros entes federativos a operacionalizar o programa federal, além de ser o município com o maior número de polos implantados no estado.

Cabe ressaltar que isso ocorreu de acordo com os preceitos das Portarias Ministeriais no 719 e no 1.402, ambas de 2011, as quais colocavam a atividade física como o eixo central do programa 2. Entretanto, no ano de 2013, o programa foi redefinido, incorporando-se o conceito de produção do cuidado e suprimindo a atividade física do objetivo geral ${ }^{3}$. Nesse contexto, a nova portaria que estabelece as diretrizes do programa retira a ênfase na promoção da atividade física, ampliando excessivamente o escopo das ações, o que pode comprometer o processo de implantação nos municípios 2 .

A implantação de uma política ou programa evidencia o quanto essa intervenção se encontra adequadamente operacionalizada 6. Segundo Hartz et al. 7, a avaliação dessa implantação permite conhecer a totalidade das intervenções quanto à validade do seu conteúdo e aos fatores que explicam possíveis defasagens entre a planificação e a execução das ações. Essa autora destaca, ainda, que tal análise demanda a construção da teoria do programa, bem como o contexto e resultados esperados.

Para atender a essas necessidades, estudos de avaliabilidade vêm sendo utilizados para apreciar em que medida um programa está em condições de ser avaliado 7 . Para Rossi \& Freeman 8 , a avaliabilidade consiste em um conjunto de procedimentos para avaliações planejadas que consideram os interesses dos envolvidos, além de maximizar a utilidade da avaliação. Eles exploram informações, expectativas e objetivos, determinando o quão avaliável esse programa é naquele momento, auxiliando na determinação dos propósitos e foco da avaliação, possibilitando compreender o programa de forma aprofundada e a identificação prévia das possibilidades de avaliação 7,9.

Estudos com abordagens tanto qualitativas quanto quantitativas têm sido utilizados no Brasil para verificar a avaliabilidade de diferentes políticas de saúde 10,11, programas de capacitação de pessoal 12 e outros programas de saúde desenvolvidos nos municípios 13,14 .

Embora programas com o propósito de oferecer atividades físicas supervisionadas e gratuitas, como é o caso do Programa Academia da Saúde, possam caracterizar-se como uma alternativa eficiente para a promoção da atividade física 15 , evidências apontam que existem poucos artigos avaliando programas de atividade física na América Latina 16. Além disso, até o momento, não existem na literatura estudos que tenham verificado a avaliabilidade do Programa Academia da Saúde.

A necessidade de verificar a avaliabilidade do Programa Academia da Saúde no Recife justifica-se por: (a) tratar-se de um programa pioneiro no Ministério da Saúde, cujas atividades se iniciam em Recife, muito antes da construção dos polos nos demais municípios brasileiros; (b) ser um programa que envolve alto investimento técnico e financeiro; (c) ter incorporado a experiência prévia do Programa Academia da Cidade cuja avaliabilidade não foi verificada anteriormente; (d) evidenciar-se a necessidade de acompanhar a implantação e reorganização do Programa Academia da Saúde.

Diante desse contexto, o objetivo do presente artigo é realizar o estudo da avaliabilidade do Programa Academia da Saúde no município do Recife, considerando a descrição da intervenção, a elaboração de seus modelos lógico e teórico de avaliação, além de identificar perguntas avaliativas. 


\section{Método}

Realizou-se um estudo avaliativo com abordagem qualitativa, valendo-se do sistema dos sete elementos proposto por Thurston \& Ramaliu 9. Esses autores entendem que um bom estudo avaliativo demanda uma estrutura capaz de assegurar êxito à análise da avaliabilidade, e que ela deve ser composta por sete passos, a saber: (1) delimitação do programa e identificação de suas metas, objetivos e atividades; (2) identificação e análise dos documentos da intervenção a ser avaliada; (3) construção do modelo lógico do programa; (4) supervisão do programa, ou entendimento preliminar de como o programa opera; (5) desenvolvimento de um modelo teórico de avaliação; (6) identificação dos usuários e envolvidos na avaliação; e (7) definição de procedimento de avaliação.

A pesquisa foi realizada no período de agosto de 2014 a junho de 2015, no Município do Recife, com o propósito de verificar a avaliabilidade do Programa Academia da Saúde.

Para a operacionalização do estudo, realizou-se uma pesquisa documental que analisou as políticas de saúde nas quais o programa se fundamenta, decretos de criação e redefinição, e as portarias que normatizam o Programa Academia da Saúde, totalizando quatro documentos 3,17,18,19.

Para a coleta dos dados, foram entrevistados 14 informantes-chave (seis gestores, dos quais dois contribuíram diretamente para a implantação do programa, e oito profissionais que atuam nos polos), identificados neste estudo conforme sua ocupação (coordenadores de 1 a 6 e professores de 1 a 8 , respectivamente), além de quatro experts no campo da avaliação de programas de saúde, todos com titulação mínima de mestre e publicações técnicas e científicas nos campos da avaliação em saúde e atividade física nos últimos cinco anos. Eles foram consultados a opinar sobre os componentes, realizar a análise lógica e o teste de consistência do modelo lógico proposto para o programa 7,20,21.

Dois instrumentos de coleta de dados foram utilizados para subsidiar a análise documental e a realização das entrevistas, que foram gravadas e transcritas na íntegra. A análise dos documentos levou em conta: (a) existência de objetivos; (b) a organização do programa; (c) público-alvo; (d) metas a serem alcançadas; (e) ações necessárias ao desenvolvimento das atividades; e (f) os resultados a serem alcançados. A análise das entrevistas seguiu um roteiro com questões relativas ao conhecimento dos objetivos do programa e à operacionalização das ações. Os gestores ainda foram perguntados acerca da necessidade e utilidade da avaliação do programa.

Visando apresentar e validar os modelos teórico e lógico do programa (propostos pelos pesquisadores e especialistas citados anteriormente), bem como discutir, identificar e pactuar as perguntas avaliativas e os procedimentos de avaliação a serem desenvolvidos em pesquisas futuras, foram realizadas duas oficinas com carga-horária de três horas, envolvendo oito profissionais e cinco dos sete gestores do programa.

As duas oficinas contaram com os mesmos participantes e aconteceram na sede administrativa do programa, sob a coordenação da pesquisadora principal deste estudo. Na primeira, os participantes receberam informações sobre o estudo (objetivo e formas de condução), o modelo lógico (utilidade e forma de construção) e o referencial normativo do programa (apresentação e discussão dos documentos do Programa Academia da Saúde). Com base nessas informações, foram apresentados (e opinaram) ao modelo lógico construído pelos autores e apreciado pelos especialistas, apresentando sugestões e solicitando esclarecimentos quando necessário. Na segunda oficina, realizou-se a apresentação do produto final dos modelos teórico e lógico e uma explanação sobre avaliação (conceitos, concepções, objetivos e aplicações). Em seguida, procedeu-se a uma discussão em grupo, guiada pelas seguintes questões: (i) que perguntas ou preocupações a respeito do programa os senhores gostariam que a avaliação respondesse?; (ii) que informações seriam úteis para administrar ou implementar melhor o programa?; e (iii) que outras informações seriam importantes?

A Tabela 1 apresenta um resumo das fontes de dados consideradas básicas e fundamentais para a consecução do estudo, e suas contribuições em cada um dos sete elementos propostos por Thurston \& Ramaliu 9.

As informações coletadas tanto na pesquisa documental quanto nas entrevistas foram organizadas em categorias e analisadas com uso da técnica da condensação de significados 22 , que analisa passagens da entrevista relacionadas a uma questão específica do estudo.

A Figura 1 apresenta a descrição das etapas do estudo de acordo com os períodos em que foram desenvolvidas. 
Tabela 1

Resumo das fontes de dados. Estudo da avaliabilidade do Programa Academia da Saúde. Recife, Pernambuco, Brasil, 2015.

\begin{tabular}{|c|c|c|}
\hline Elemento & Objetivos & Fontes de dados \\
\hline 1 & $\begin{array}{l}\text { Analisar a normativa do programa a respeito de sua descrição, metas, objetivos e } \\
\text { atividades, e identificar a percepção dos atores acerca desses componentes. }\end{array}$ & $\begin{array}{l}\text { Portaria no } 2.681 / 20133 \\
\text { Entrevistas com gestores e professores. }\end{array}$ \\
\hline 2 & $\begin{array}{l}\text { Analisar a adequação, contexto, conceitos-chave, factibilidade, coerência e viabilidade } \\
\text { dos documentos normativos do Programa Academia da Saúde. }\end{array}$ & $\begin{array}{l}\text { Portaria no 2.681/2013 3; } \\
\text { Política Nacional de Promoção da Saúde 17; } \\
\text { Decreto no 19.808/2003 18; } \\
\text { Política Nacional de Atenção Básica } 19 \text {. }\end{array}$ \\
\hline 3 & Construir e validar o modelo lógico do programa & $\begin{array}{l}\text { Portaria no } 2.681 / 2013 \text {; } \\
\text { Consulta a especialistas; } \\
\text { Oficina com profissionais e gestores. }\end{array}$ \\
\hline 4 & Descrever a organização e funcionamento do programa & $\begin{array}{l}\text { Portaria no } 2.681 / 2013 \text { 3; } \\
\text { Decreto no } 19.808 / 2003 \text { 18; } \\
\text { Entrevistas com gestores e professores. }\end{array}$ \\
\hline 5 & $\begin{array}{l}\text { Descrever a teoria do programa e identificar as relações entre os componentes do } \\
\text { modelo lógico, os atores e o contexto de implantação. }\end{array}$ & $\begin{array}{l}\text { Portaria no } 2.681 / 2013 \text { 3; } \\
\text { Decreto no } 19.808 / 2003 \text { 18; } \\
\text { Entrevistas com gestores e professores. }\end{array}$ \\
\hline 6 & Identificar os usuários e envolvidos na avaliação. & Entrevistas com gestores e professores. \\
\hline 7 & Identificar e pactuar o procedimento de avaliação a ser realizado em estudo posterior. & Oficina com profissionais e gestores. \\
\hline
\end{tabular}

\section{Figura 1}

Etapas do estudo da avaliabilidade do Programa Academia da Saúde. Recife, Pernambuco, Brasil, 2015.

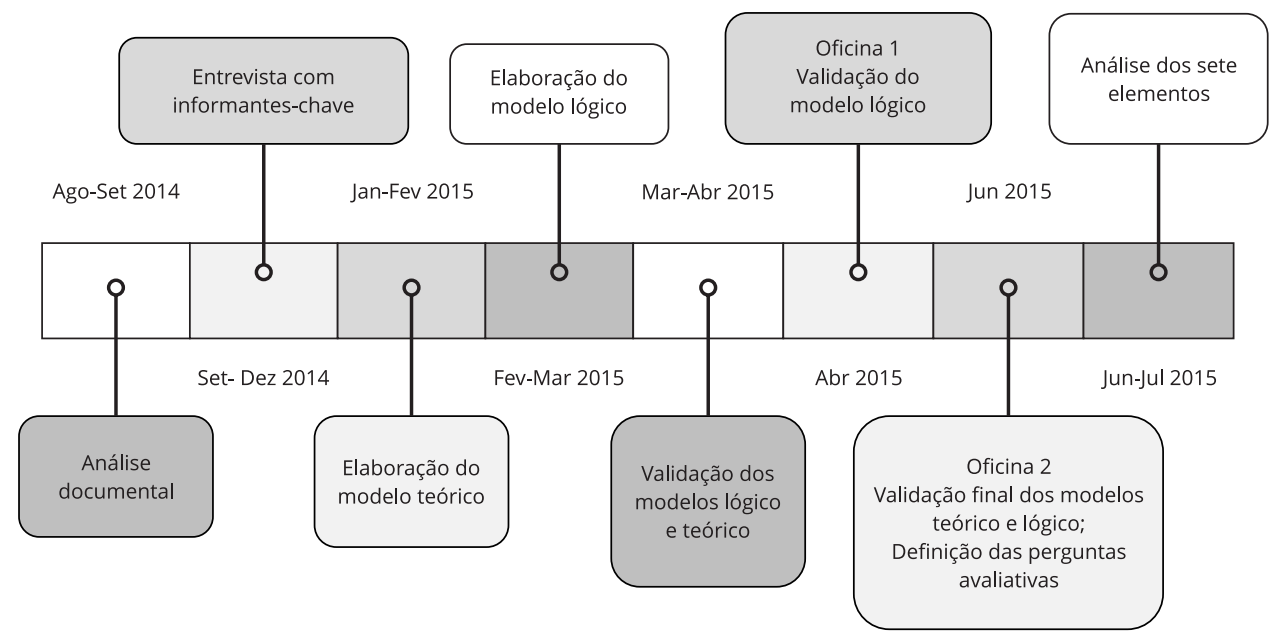

Este estudo foi aprovado pelo Comitê de Ética em Pesquisas do Centro de Ciências da Saúde da Universidade Federal de Pernambuco, parecer no 818.708. 


\section{Resultados}

A Tabela 2 apresenta os resultados do estudo da avaliabilidade do Programa Academia da Saúde no Recife, segundo os sete elementos propostos por Thurston \& Ramaliu 9.

\section{Elemento 1 - delimitação do programa e identificação de suas metas, objetivos e atividades}

O Programa Academia da Saúde surge no Município do Recife pela incorporação de 37 polos do Programa Academia da Cidade, que fora instituído desde 2002, com o objetivo de implementar e desenvolver ações que contemplassem a prática de atividade física regular, de forma orientada e sistematizada, visando à promoção da saúde da população em suas diversas faixas etárias, com utilização de espaços públicos do Recife 18.

Para que o Programa Academia da Cidade se incorporasse ao Programa Academia da Saúde, foi necessário que o programa preexistente passasse a cumprir as diretrizes estabelecidas nas Portarias no 2.681 e no 2.684 de 2013. Elas estabelecem que o Programa Academia da Saúde deve ser composto por polos que se configuram como espaços públicos destinados às ações do programa e que suas atividades devem desenvolver-se em oito eixos, em conformidade com seis princípios, três diretrizes e 14 objetivos específicos.

Verificou-se que as diretrizes do Programa Academia da Saúde descrevem como objetivo do programa a ampliação do escopo das ações de promoção da saúde, no entanto não definem claramente as metas para o alcance de tais objetivos, nem o público-alvo das ações do programa. Entretanto, os objetivos, eixos e diretrizes denotam o direcionamento das metas aos profissionais (apoio às ações de promoção da saúde e integralidade do cuidado) e usuários dos serviços de saúde (identificação como um ponto de atenção para ações de promoção, empoderamento e controle social), especialmente na atenção primária à saúde.

Cabe ressaltar que as entrevistas com os informantes-chave apontam divergências na percepção dos objetivos do Programa Academia da Saúde entre professores e coordenadores. Os últimos demonstram não conhecer os objetivos propostos nas portarias normativas, as quais ampliam o escopo da promoção da saúde para além da atividade física, descrevendo apenas a oferta delas como objetivos do programa, conforme se constata nos seguintes depoimentos:

"O objetivo do programa é ofertar a prática regular supervisionada, orientada para a população, o incentivo da prática de atividade física de forma gratuita..." (coordenador 3).

"O principal é trabalhar a questão da atividade física, trabalhar a promoção de saúde através da atividade física..." (coordenador 2).

Por outro lado, os profissionais que atuam nos polos conhecem os objetivos do programa, descrevendo a promoção da saúde como centro das intervenções:

"Promover a saúde dos alunos, da melhor forma possivel para que eles se sintam bem com eles mesmos..." (professor 1).

"O objetivo é promoção da saúde e também a disseminação de práticas corporais, de atividades físicas nos espaços públicos de modo a contribuir com a promoção da saúde do usuário" (professor 8).

Da mesma forma que as metas e o público-alvo, as atividades necessárias ao desenvolvimento do Programa Academia da Saúde não são claramente definidas. Porém, a Portaria no 2.681 estabelece que elas devem acontecer em conformidade com oito eixos temáticos, a saber: (a) práticas corporais e atividades físicas; (b) produção do cuidado e de modos de vida saudáveis; (c) promoção da alimentação saudável; (d) práticas integrativas e complementares; (e) práticas artísticas e culturais; (f) educação em saúde; (g) planejamento e gestão; e (h) mobilização da comunidade.

\section{Elemento 2 - identificação e análise dos documentos do Programa Academia da Saúde}

Para essa etapa, foram analisados os documentos que regem o Academia da Saúde, a saber: (a) portarias que redefiniram o Programa Academia da Saúde em 2013 3,23; (b) a Política Nacional de Atenção Básica 17; (c) a Política Nacional de Promoção da Saúde 19; e (d) o Decreto Municipal no 19.808 de 3 de abril de 2003, que institui o Programa Academia da Cidade em Recife (precursor do Programa Academia da Saúde no município) 18. 


\section{Tabela 2}

Resultados do estudo da avaliabilidade do Programa Academia da Saúde, segundo os sete elementos de Thurston \& Ramaliu 9. Recife, Pernambuco, Brasil, 2015.

\section{Elementos}

(1) Metas, objetivos e atividades que constituem o programa;

(2) Revisão dos documentos;

(3) Modelagem dos recursos disponíveis, programa de atividades pretendidas, impactos esperados e conexões causais presumidas;

(4) Supervisão do programa, ou entendimento preliminar de como o programa opera;

(5) Desenvolvimento de um modelo de programa avaliável; impactos esperados e conexões causais presumidas;

(6) Identificação de usuários envolvidos na avaliação e outros principais envolvidos;

(7) Obtenção de um acordo quanto ao procedimento de uma avaliação.
Resultado

Metas: os documentos oficiais não estabelecem metas para o Programa Academia da Saúde; Não há consenso sobre as metas do programa entre os profissionais entrevistados e alguns deles confundem metas com objetivos do programa.

Objetivo: contribuir para a promoção da saúde e produção do cuidado e de modos de vida saudáveis da população pela implantação de polos com infraestrutura e profissionais qualificados.

Atividades: o programa prevê o desenvolvimento das atividades em oito eixos em conformidade com três diretrizes, seis princípios e 14 objetivos específicos.

Foram identificados e analisados quatro documentos que tratam dos objetivos, princípios e diretrizes do programa, porém se mostram pouco esclarecedores para a operacionalização das ações.

A descrição do programa explicitando seus componentes, atividades e efeitos é apresentada no modelo lógico do programa, ao longo do texto.

Realização de sessões de atividades físicas nos polos e Centros de Apoio Psicossocial em dois turnos nos dias úteis. Desenvolvimento de ações pontuais de educação em saúde.

Elaboração de um modelo teórico, informando como se espera que o programa exerça suas atividades no território, contemplando sua estrutura organizacional e a existência de dispositivos formais que regulamentam o processo de avaliação.

Foram identificados a população, o secretário de saúde do município e os gestores da atenção primária, além dos gestores e profissionais do programa.

Definiu-se que a avaliação será realizada mediante desenvolvimento de estudo de caso, visando verificar o grau de implantação do programa e os elementos do contexto que interferem nesse grau de implantação.

Esses documentos apresentam coerência com o contexto de reorganização das ações e serviços no âmbito do Sistema Único de Saúde (SUS), fundamentam-se e articulam diretrizes e conceitos da atenção primária à saúde e da promoção da saúde. Por outro lado, descrevem uma ampla gama de objetivos específicos que devem ser desenvolvidos em diferentes eixos e diretrizes.

\section{Elemento 3 - construção do modelo lógico do Programa Academia da Saúde}

De acordo com a análise documental e das entrevistas com os informantes-chave elaborou-se o modelo lógico do Programa Academia da Saúde em Recife (Figura 2), que objetiva identificar os recursos, atividades, e efeitos esperados com a implantação do programa (resultados parciais e finais), além de buscar a identificação de uma cadeia causal de premissas que articula tais elementos.

Foram definidos três componentes (gestão, atenção à saúde nas linhas de cuidado e promoção da saúde) e 11 subcomponentes, conforme proposto pelos especialistas consultados.

\section{Elemento 4 - entendimento preliminar de como o programa opera}

O relato dos gestores revela que o programa opera em dois turnos (manhã e noite), fundamentalmente pelo desenvolvimento de atividades físicas/práticas corporais orientadas por profissionais de educação física, tanto nos polos, quanto no território. Os conteúdos mais presentes nas atividades são: ginástica, dança, jogos, passeios e rodas de conversa: 
Figura 2

Modelo lógico do Programa Academia da Saúde. Recife, Pernambuco, Brasil, 2015.

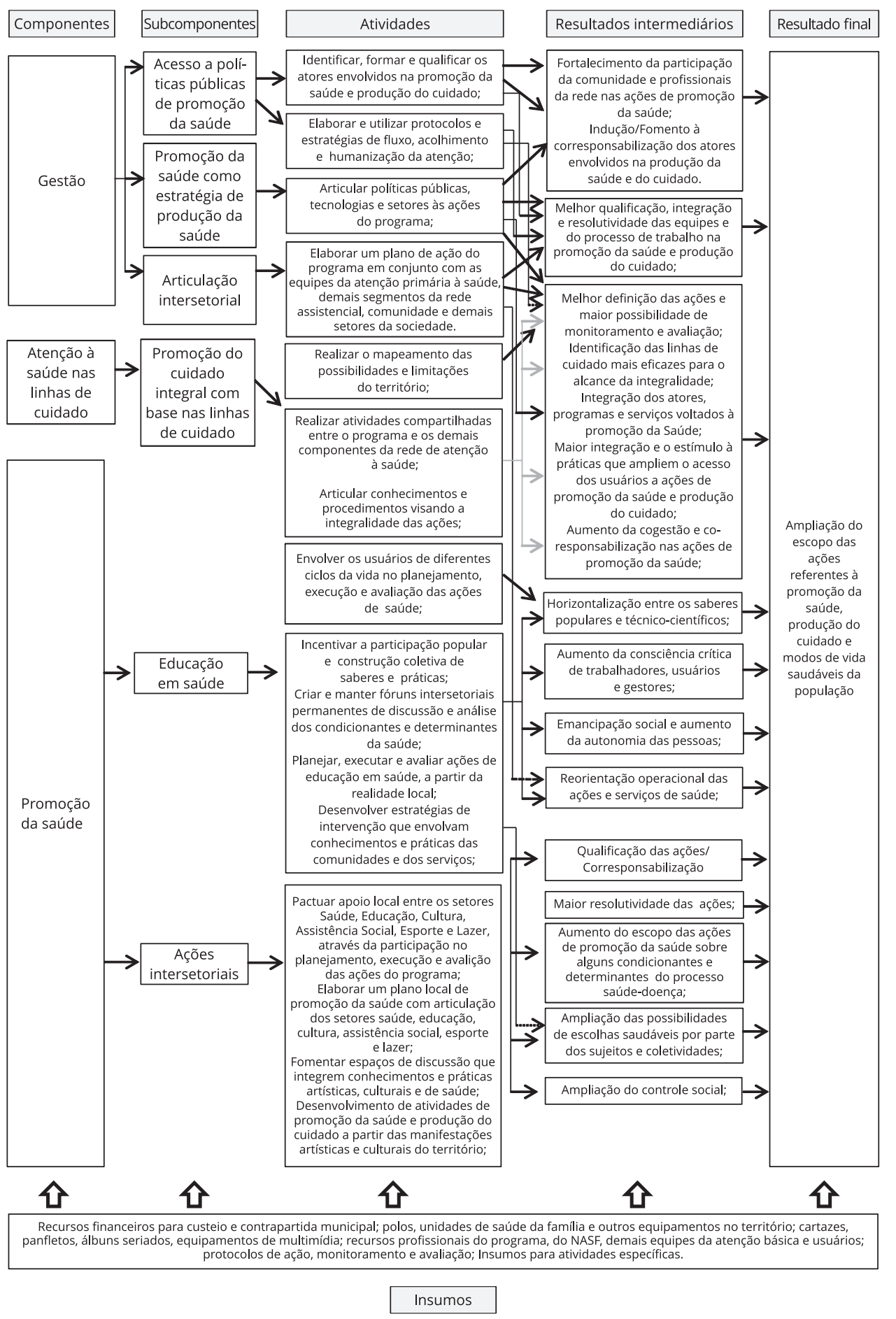

(continua) 
Figura 2 (continuação)

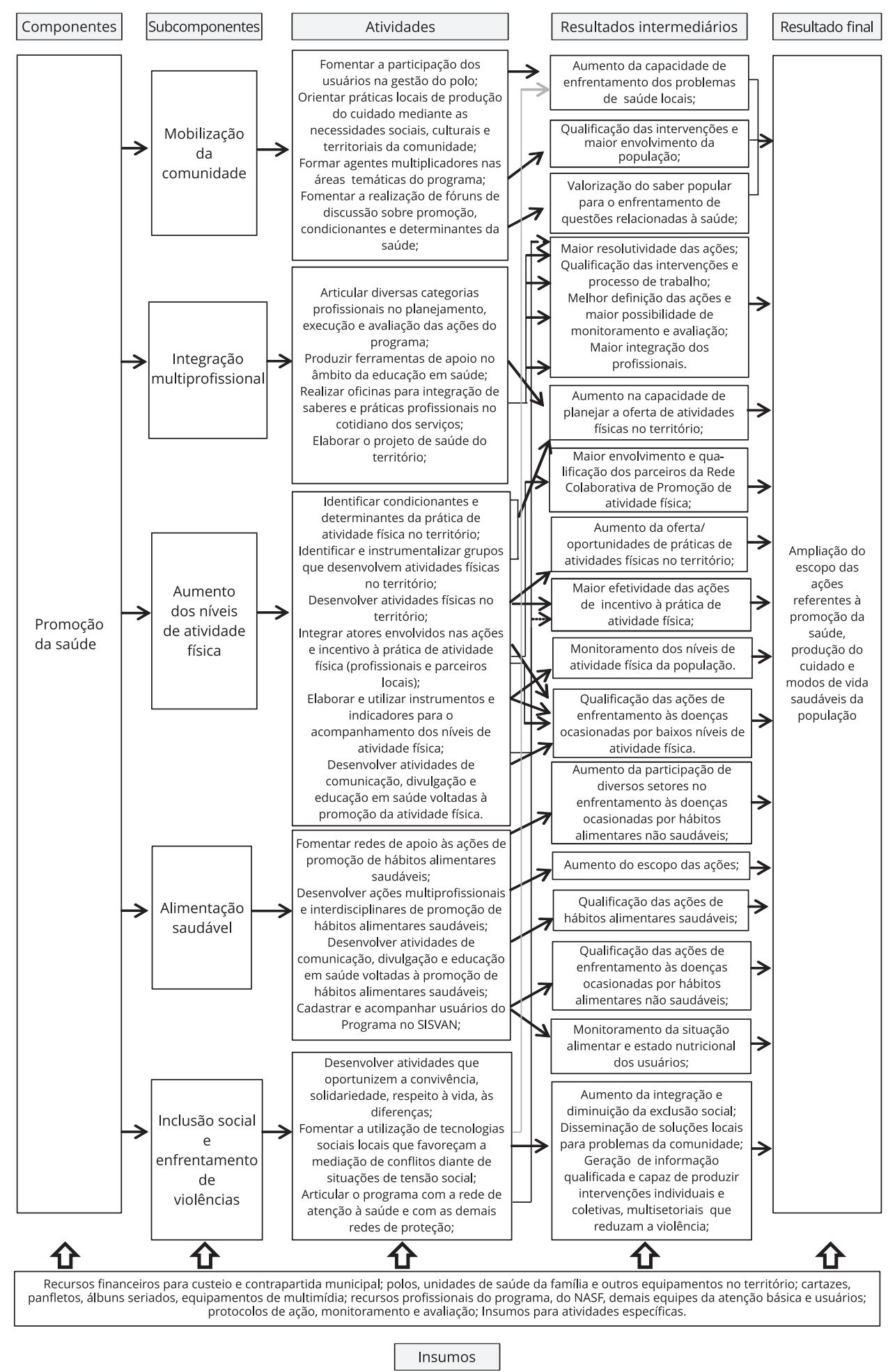

NASF: Núcleo de Apoio à Saúde da Família; SISVAN: Sistema de Vigilância Alimentar e Nutricional. 
"Nosso programa ele tem várias ações, né? A gente atua primariamente nos polos...então nós temos equipes diurnas e noturnas, né? Então esse profissional ele atua nesses locais com a prática de atividade física por orientação profissional, com orientação na área da saúde, integração social, né..." (coordenador 2)

“...Somos profissionais de nível superior, com a carga horária dividida em três horas no polo, e a que a gente diz ser extrapolo: que aí pode ser um grupo de idosos, ou grupo de alguma comunidade, que pode ser acompanhado por um agente comunitário de saúde, pode ser o Centro de Atenção Psicossocial ou ainda pode ser dentro de uma unidade de saúde..." (coordenador 1$)$.

"Nos polos a gente trabalha além da questão da atividade física propriamente dita a parte de ginástica, avaliações físicas, rodas de diálogo, passeios, jogos, dança...” (coordenador 5).

"O usuário vai encontrar aulas que estão dentro da cultura corporal: ginástica, luta... você vai ver luta, ginástica, dança...” (coordenador 3).

Os gestores relatam que, além das práticas corporais, o programa desenvolve parcerias com unidades de saúde, contudo a descrição delas não demonstra continuidade das ações e denota um caráter pontual, focado em atividades de educação em saúde desenvolvidas em datas festivas, ou em acordo com o calendário da saúde:

“...Procuramos fazer nosso trabalho também interligado às unidades de saúde. Então, obedecendo também, norteando também, obedecendo e se norteando pelo calendário de saúde anual..." (coordenador 5).

"Bom, hoje o programa desenvolve além da prática corporal, né?... a gente tem parcerias com a unidade de saúde, né, com o Núcleos de Apoio à Saúde da Família [NASF]; então a gente tem os trabalhos de educação em saúde, né? Tem os eventos... principais durante o ano, né, mais focado é o dia mundial da saúde; quando tem o carnaval, tem atividade focada no carnaval, São João... sempre procurando focar na agenda da saúde, né? É no calendário da saúde que a gente procura trabalhar! Em relação a esse calendário...” (coordenador 4).

“...as rodas de diálogos, com os momentos de educação em saúde, praticamente todos os meses acontecem essas rodas de diálogos, com temas diversos...” (coordenador 1).

\section{Elemento 5 - desenvolvimento do modelo teórico de avaliação}

O modelo teórico apresentado na Figura 3 descreve a teoria do programa, além de identificar e apontar as relações entre os componentes do modelo lógico, o contexto (político-institucional e externo) e as ações desenvolvidas (cenário de implantação) para o Programa Academia da Saúde no Recife alcançar os objetivos esperados.

Os componentes gestão e atenção à saúde nas linhas de cuidado (do modelo lógico) demandam ações no sistema de saúde e do poder público municipal, sendo influenciados especialmente pelos elementos do contexto político-institucional. O componente promoção da saúde sofre influência de ambos os contextos, mas demanda um maior aporte de ações que envolvem atores de outros pontos da rede de atenção à saúde, usuários e articulações intersetoriais.

A dimensão teórica de um programa fundamenta-se, entre outras coisas, na decisão política que antecede a sua operacionalização e é composta: (a) pelo problema que demandou a criação da intervenção; (b) pelo contexto/cenário em que ela se desenvolve; e (c) pelos atores evolvidos no seu planejamento e execução.

\section{- Problema}

A análise dos documentos que normatizam o Programa Academia da Saúde aponta que o programa foi desenvolvido para ampliar o escopo e a articulação das ações de promoção da saúde na atenção primária à saúde. Entretanto, as entrevistas com informantes-chave revelam a dificuldade de articulação com outras ações e serviços nesse nível de atenção, conforme os discursos de um gestor e de um professor:

"A gente sabe que... a parceria com as outras políticas públicas do município é importante mas... a gente parte das atividades físicas! atividade corporal!" (coordenador 6).

“...Não tem uma articulação! As unidades de saúde da família, é... tem algumas próximas daqui, mas a gente não consegue ter nenhum tipo de articulação com elas" (professor 4). 
Figura 3

Modelo Teórico de Avaliação do Programa Academia da Saúde. Recife, Pernambuco, Brasil, 2015.

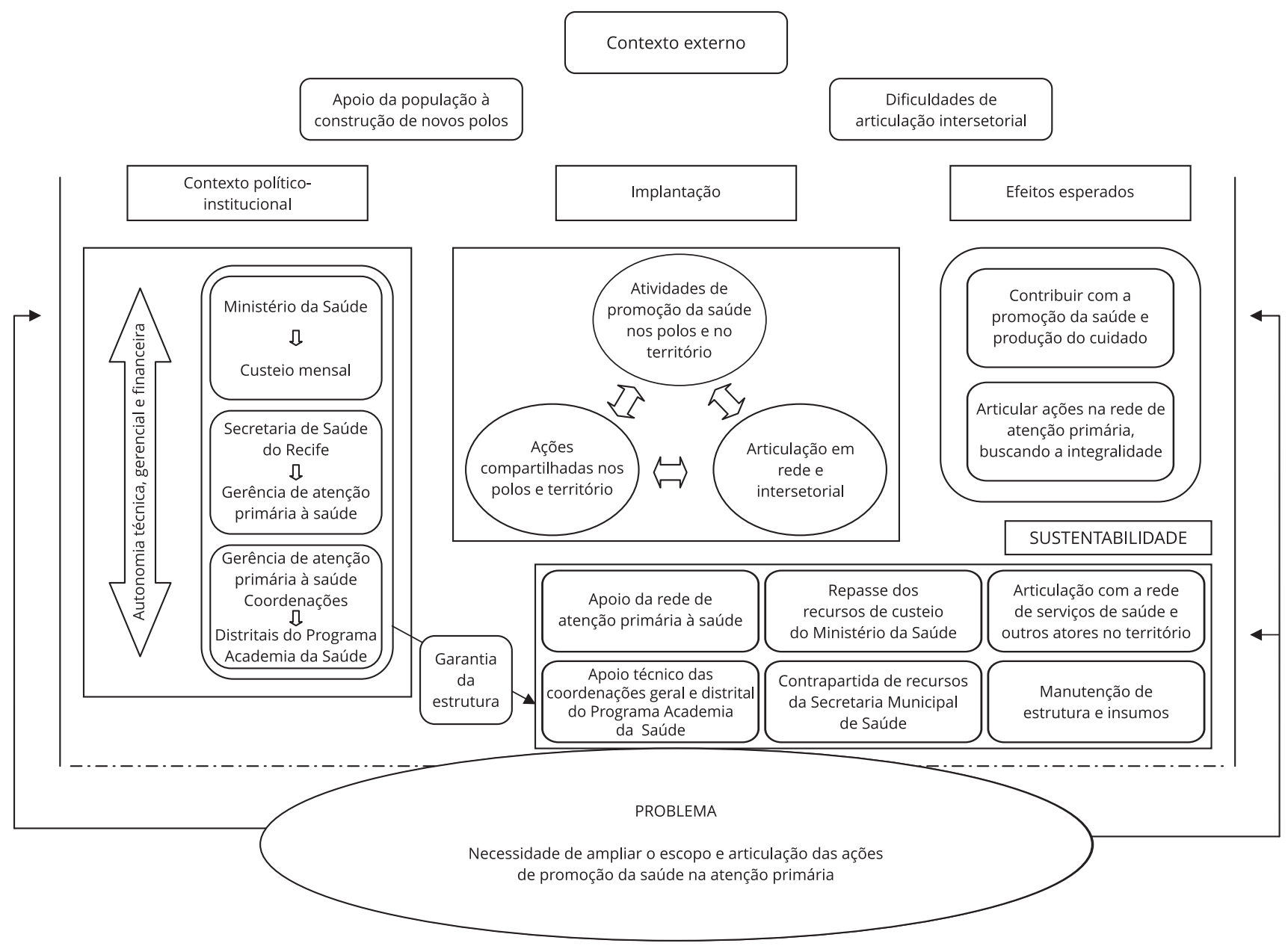

\section{- Contexto/cenário}

O Programa Academia da Saúde foi implantado no Recife pela incorporação de polos já existentes do Programa Academia da Cidade que atendiam aos critérios de similaridade ao programa federal para o recebimento dos recursos de custeio. Seu cenário de implantação se caracteriza pela execução de atividades físicas nos polos, Centros de Apoio Psicossocial (CAPS) e em algumas unidades de saúde da família, que são complementadas por ações pontuais de educação em saúde.

Entre os elementos favoráveis do contexto político-institucional, as entrevistas apontam a criação do cargo de profissional de educação física na saúde, a realização de concurso público para provimento desses postos de trabalho no âmbito da Secretaria Municipal de Saúde, o investimento financeiro da gestão local e a busca por parcerias junto ao governo estadual para a construção de novos polos do Programa Academia da Cidade que posteriormente se incorporaram ao Programa Academia da Saúde. 
Verificou-se a participação dos usuários como elemento favorável do contexto externo. Eles participam da gestão do Programa Academia da Saúde indicando os locais para a construção de polos nas reuniões do orçamento participativo do Recife.

Por outro lado, a análise documental do programa bem como as entrevistas com gestores e profissionais apontam que as diretrizes e normativas imprecisas, ausência de protocolos de ação, falta de articulação com as demais ações e serviços na atenção primária, falta de investimento financeiro e dificuldades de manutenção das estruturas físicas e do material são elementos do contexto que influenciam negativamente no desenvolvimento das atividades e na implantação do programa.

\section{- Atores}

Os atores da construção do Programa Academia da Saúde no Recife são os gestores, trabalhadores e a população que, em diferentes espaços e momentos, contribuíram para a implantação do programa nos contextos apresentados acima.

\section{Elementos 6 e 7 - identificação dos usuários e definição dos procedimentos de avaliação}

Os professores e gestores que participaram da segunda oficina sobre a avaliabilidade do Programa Academia da Saúde apontaram que os principais usuários e envolvidos na avaliação do programa seriam a população, o secretário de saúde do município, os gestores da atenção primária à saúde, além dos gestores e profissionais do programa.

Nesse sentido, ainda durante a oficina, as perguntas avaliativas foram formuladas mediante análise de questões sobre a avaliação do programa contidas nas entrevistas (fase divergente) e da apresentação e pactuação delas e dos modelos teórico e lógico (fase convergente).

No que cabe à utilidade da avaliação, os gestores consideram que ela pode contribuir para a verificação do cumprimento das diretrizes, do alcance dos objetivos do programa e para subsidiar a tomada de decisão com base no acompanhamento e adequação das ações realizadas.

Ao final dessa oficina, definiu-se que a avaliação do programa deveria ser realizada por estudo de caso, contemplando as perguntas: (a) qual o grau de implantação do Programa Academia da Saúde no Recife? (b) os elementos dos contextos político e externo influenciaram na implantação do programa? (c) de que maneira? (d) o grau de implantação do Programa Academia da Saúde influencia nos efeitos? (e) de que maneira?

\section{Discussão}

Este estudo de avaliabilidade foi conduzido no intuito de descrever o Programa Academia da Saúde, identificando seus objetivos e metas. Além disso, foram elaborados e validados os seus modelos lógico e teórico e definidas as perguntas avaliativas para avaliação formativa.

Foi realizado por avaliadores externos, com a participação e apoio de especialistas em avaliação, profissionais e gestores do programa, o que segundo Thurston \& Ramaliu 9 permite que sejam promovidas mudanças no programa desde os primeiros achados da avaliação. Além disso, a participação de diferentes atores favorece a interpretação dos resultados, que passam a servir a todos os usuários, possibilitando a reorientação das ações na busca pelos objetivos previstos 25 .

A pesquisa documental selecionou os documentos que permitiam a identificação dos princípios, objetivos e metas do programa. Os dois primeiros são descritos com clareza, embora, ao confrontar os objetivos propostos nos instrumentos normativos com a percepção dos informantes-chave, se verifique que só o discurso dos professores se aproxima do descrito nos documentos. Por outro lado, a percepção dos coordenadores sobre os objetivos do programa diverge dos descritos nas diretrizes do Programa Academia da Saúde. Tal divergência de entendimentos sobre os objetivos pode estar associada à redefinição das diretrizes Programa Academia da Saúde, em 2013 (dois anos após a implantação no Recife), que retirou do objetivo geral do programa a ênfase da promoção da atividade física e ampliou excessivamente seu escopo de ações, comprometendo a identificação do seu foco principal 2. Por outro lado, de acordo com Leviton et al. 26, a identificação de divergências entre a percepção dos 
envolvidos e os objetivos propostos para a intervenção é comum em estudos de avaliabilidade, porém esse desconhecimento dos objetivos compromete a operacionalização das ações e aponta a necessidade de avaliação dessa área específica do programa.

De acordo com Natal et al. ${ }^{12}$, programas em fase de criação tendem a apresentar poucos documentos relativos à organização e funcionamento. No caso do Programa Academia da Saúde, isso pode estar relacionado ao fato de tratar-se de um programa relativamente novo cuja implantação só se iniciou de fato em municípios que conseguiram incorporar iniciativas preexistentes de acordo com critérios de similaridade, muito antes do início das obras para a construção de polos nas demais unidades da federação. À medida que esses programas avançam, diferentes tipos de documentos podem ser elaborados de acordo com a sua dimensão e complexidade 12. O Ministério da Saúde parece estar avançando nesse sentido, pela publicação de um manual sobre infraestrutura dos polos do programa 27 e do anúncio da publicação de uma série de outros visando à operacionalização das ações 28 .

No que concerne à organização do programa, verifica-se que as diretrizes descrevem uma proposta audaciosa, com uma ampla gama de ações a serem desenvolvidas em oito eixos, e em conformidade com seis princípios, três diretrizes e 14 objetivos específicos. Esse escopo de ações demanda uma grande capacidade de articulação em "redes de compromisso e responsabilidade", além do envolvimento de diversos atores, o que compromete a factibilidade e torna difícil a operacionalização 29 .

Os documentos oficiais não definem as metas, nem os resultados esperados pela implantação do programa, além de não descreverem claramente as ações necessárias ao desenvolvimento do Programa Academia da Saúde. Todavia, a definição dos eixos, princípios e diretrizes permite o direcionamento aos preceitos estabelecidos nas políticas nacionais de atenção básica 19 e de promoção da saúde 17 , com finalidade de ampliação do escopo das ações de promoção da saúde e produção do cuidado, com implícitas possibilidades de adequação das ações à realidade local.

Modelos lógicos de avaliação permitem realizar uma descrição essencial de intervenção 20,30 e contribuem para a identificação dos componentes de um programa e das conexões entre eles e os resultados esperados 20,25. O modelo lógico do Programa Academia da Saúde no Recife foi elaborado tendo em conta três componentes: gestão; atenção à saúde nas linhas de cuidado; promoção da saúde. A escolha da categoria gestão levou em consideração que ela é fundamental para a operacionalização do programa, pois demanda uma série de ações político-administrativas voltadas ao planejamento, acompanhamento de processos, resultados e avaliação de ações com intuito da tomada de decisão em uma determinada instituição 31 . Os demais componentes foram definidos mediante arrolamento das atividades do programa, descritos nos documentos oficiais. Os subcomponentes foram extraídos dos objetivos específicos do programa, conforme previsto na literatura 31.

A sistematização do modelo foi realizada com base nos insumos (estrutura) e atividades (processos) para os resultados esperados, visando descrever o caminho causal para que as ações do Programa Academia da Saúde gerassem os resultados esperados 20.

Com relação ao entendimento preliminar de como o programa opera, verificou-se que as ações ocorrem fundamentalmente nos polos, em dois turnos e sob a supervisão de profissionais de nível superior (educação física), conforme previsto nas diretrizes do programa ${ }^{3}$. As atividades mais desenvolvidas são os exercícios físicos, jogos, passeios e rodas de conversa. Essas mesmas rotinas são descritas em outros estudos sobre programas comunitários de atividades físicas 32,33 . Os informantes-chave ainda relatam o desenvolvimento de ações de educação em saúde em datas festivas, denotando um caráter pontual e possível perda da continuidade dessas intervenções. Estudos realizados por Radlley 34 e Alencar et al. 35 demonstram que ações pontuais de educação em saúde são pouco efetivas para alterar comportamentos relacionados à saúde e fragilizam a prática profissional.

A construção do modelo teórico e da delimitação da teoria do programa demandou a compreensão de que o Programa Academia da Saúde surgiu no Recife, nove anos após a implantação e funcionamento do Programa Academia da Cidade 3,18, cujos objetivos originais e estratégias de operação diferem das previstas para o Academia da Saúde, sobretudo após a sua redefinição em 2013, no que se refere ao problema que se pretendia resolver, aos objetivos gerais e escopo das ações. Enquanto o precursor municipal destinava-se à requalificação de espaços públicos de lazer e oferta de atividades físicas orientadas 18, o sucessor almeja contribuir para a promoção da saúde numa perspectiva voltada à ampliação e articulação de ações no âmbito da atenção primária, integrando diferentes atores e saberes, com fito de configuração e consolidação de uma rede assistencial 3,19 . 
A elaboração da teoria do programa e de sua modelagem (elaboração dos modelos lógico e teórico) buscou identificar e descrever o estágio atual de desenvolvimento do programa no Recife. Esse processo torna mais clara a teoria do programa e permite a elaboração de perguntas avaliativas 20,25,36.

A pactuação de critérios e padrões de julgamento entre os interessados na avaliação permite elaborar estratégias avaliativas orientadas pelos resultados 22,25. Nesse sentido, a oficina para definição dos procedimentos de avaliação revelou o interesse dos trabalhadores e gestores do Programa Academia da Saúde em identificar o grau de implantação do programa no Recife e analisar as potenciais influências dos elementos dos contextos político e externo na implantação do programa, além de verificar se o grau de implantação influencia nos efeitos e de que maneira. Essas perguntas avaliativas se assemelham às identificadas por Oliveira et al. 38 ao realizarem o estudo avaliativo do Programa de Controle da Tuberculose do Sistema Penitenciário de dois estados brasileiros.

\section{Considerações finais}

Este estudo aponta a viabilidade metodológica e operacional para o desenvolvimento de uma pesquisa avaliativa, além de identificar as necessidades e estratégias para a realização dessa atividade, em consonância com os interesses dos atores envolvidos.

Verificou-se que as diretrizes do programa são muito abrangentes no que tange às possibilidades de ação, porém pouco esclarecedoras no que se refere ao planejamento, operacionalização e avaliação das ações, devendo ser aperfeiçoadas a fim de contribuir de forma mais efetiva para o processo de implantação do Programa Academia da Saúde nos municípios e no Distrito Federal.

A modelagem descrita neste estudo avaliativo buscou definir uma "imagem-objetivo" do Programa Academia da Saúde no Recife, objetivando subsidiar a apreciação do grau de implantação dos seus componentes, a ser realizada em futuros estudos, seja para verificar a operacionalização dos seus processos, seja para mensurar o alcance dos objetivos pretendidos.

Os resultados deste trabalho podem contribuir para a realização de novos estudos de avaliabilidade em outros programas e políticas de saúde, além de subsidiar a readequação das atividades e elaboração de normas operacionais mais claras que facilitem o processo de implantação e operacionalização das ações, bem como a avaliação sistemática dessas nas três esferas de gestão do SUS.

Como limitação deste estudo, aponta-se a dificuldade de mobilizar um número maior de informantes-chave para as entrevistas, construção e validação do modelo lógico do programa. Por outro lado, verifica-se como ponto forte a forma didática como o sistema dos sete passos foi descrito, facilitando a replicação desta pesquisa, tanto para estudos de avaliabilidade do Programa Academia da Saúde, quanto para outras ações e programas de saúde.

Entre os desafios que se apresentam, destaca-se a necessidade de realizar estudos de avaliabilidade nos demais entes federativos que implantaram o programa, além da verificação do impacto e da viabilidade econômica do Programa Academia da Saúde. 


\section{Colaboradores}

R. N. Silva, F. R. B. Guarda, P. C. Hallal e P. J. L. Martelli contribuíram na concepção e projeto, análise e interpretação dos dados; redação do artigo, revisão crítica relevante do conteúdo intelectual; aprovação final da versão a ser publicada.

\section{Agradecimentos}

Agradecemos às professoras Juliana Martins Barbosa da Silva Costa, Luciana Santos Dubeux, Louisiana Regadas de Macedo Quinino, Mariana Sena Barreto de Melo, Júlia Aparecida Devidé Nogueira, Adriana Falângola Benjamin Bezerra, Albanita Gomes C. Ceballos e Maria Luiza Timóteo pelas contribuições prestadas tanto para na validação do modelo lógico, quanto na crítica ao trabalho que originou este manuscrito. Agradecemos também, à Secretaria de Saúde do Recife, por ter colaborado com a realização deste estudo.

\section{Referências}

1. Knuth AG, Malta DC, Cruz DK, Castro AM, Fagundes J, Sardinha LM, et al. Description of the countrywide physical activity network coordinated by the Brazilian Ministry of Health: 2005-2008. J Phys Act Health 2010; 7 Suppl 2:S253-8.

2. Hallal PC. Atividade física e saúde no Brasil: pesquisa, vigilância e políticas. Cad Saúde Pública 2014; 30:2487-9.

3. Ministério da Saúde. Portaria no 2.681, de 7 de novembro de 2013. Redefine o Programa Academia da Saúde no âmbito do Sistema Único de Saúde (SUS). Diário Oficial da União 2013; 8 nov.

4. Malta DC, Silva MMA, Albuquerque GM, Lima CM, Cavalcante T, Jaime PC, et al. A implementação das prioridades da Política Nacional de Promoção da Saúde, um balanço, 2006 a 2014. Ciênc Saúde Coletiva 2014; 19:4301-12.

5. Simões EJ, Hallal P, Pratt M, Ramos L, Munk M, Damascena W, et al. Effects of a community-based, professionally supervised intervention on physical activity levels among residents of Recife, Brazil. Am J Public Health 2009; 99:68-75.

6. Leviton LC, Khan LK, Rog D, Dawkins N, Cotton D. Evaluability assessment to improve public health policies, programs, and practices. Ann Rev Public Health 2010; 31:213-33.

7. Hartz ZMA, Vieira-da-Silva LM. Avaliação em saúde: dos modelos teóricos à prática na avaliação de programas e sistemas de saúde. Rio de Janeiro: Editora Fiocruz; 2005.

8. Rossi PH, Freeman HE. Evaluation: a system approach. Newbury Park: Sage Publications; 1989.

9. Thurston W, Ramaliu A. Evaluability assessment of a survivors of torture program: lessons learned. Can J Program Eval 2005; 20:1-25.

10. Bezerra LCA, Alves CKA, Reis YAC, Samico I, Felisberto E, Carvalho ALB, et al. Identificação e caracterização dos elementos constituintes de uma intervenção: pré-avaliação da política ParticipaSUS. Ciênc Saúde Coletiva 2012; 17:883-900.

11. Nascimento LV, Machado WD, Gomes DF, Vasconcelos MIO. Estudo de avaliabilidade da Política Nacional de Atenção Integral à Saúde do Homem no município de Sobral, Ceará Rev Baiana Saúde Pública 2014; 38:95-114.

12. Natal S, Samico I, Oliveira RGD, Assis AMJ. Estudo de avaliabilidade da rede de formação de Recursos Humanos da Secretaria de Vigilância em Saúde do Ministério da Saúde. Cad Saúde Colet (Rio J.) 2010; 18:560-71.

13. Dhalia CBC, Díaz-Bermúdez XP. Teste rápido - por que não?: estudos que contribuíram para a política de ampliação da testagem para o HIV no Brasil. Brasília: Ministério da Saúde; 2007.

14. Medeiros PFP, Bezerra RCA, Santos NTV, Melo EO. Um estudo sobre a avaliabilidade do Programa + Vida: política de redução de danos em álcool, fumo e outras drogas do município de Recife, Brasil. Rev Bras Saúde Matern Infant 2010; 10 Suppl:s209-s17. 
15. Mendonça BC, Oliveira AC, Toscano JJO, Knuth AG, Borges TT, Malta DC, et al. Exposure to a community-wide physical activity promotion program and leisure-time physical activity in Aracaju, Brazil. J Phys Act Health 2010; 7 Suppl 2:S223-8.

16. Hoehner CM, Soares J, Parra Perez D, Ribeiro IC, Joshu CE, Pratt M, et al. Physical activity interventions in Latin America: a systematic review. Am J Prev Med 2008; 34:224-33.

17. Secretaria de Vigilância em Saúde, Secretaria de Atenção à Saúde, Ministério da Saúde. Política Nacional de Promoção da Saúde - PNPS: revisão da Portaria MS/GM no 687, de 30 de março de 2006. Brasília: Ministério da Saúde; 2015.

18. Prefeitura Municipal do Recife. Decreto no 19.808, de 03 de abril de 2003. Institui e regulamenta o Programa Academia da Cidade http://www.legiscidade.recife.pe.gov.br/de creto/19808/ (acessado em 25/Mar/2015).

19. Ministério da Saúde. Política Nacional de Atenção Básica. Brasília: Ministério da Saúde; 2012. (Série Pactos pela Saúde, 4).

20. U.S. Department of Health and Human Services. Handbook for evaluation of physical activity programs in Latin America. http:// www.cdc.gov/nccdphp/dnpa (acessado em 25/ Mar/2015).

21. Ferreira H, Cassiolato M, Gonzalez R. Como elaborar modelo lógico de programa: um roteiro básico. Nota técnica. Brasília: Instituto de Pesquisa Econômica Aplicada; 2007.

22. Kvale S. Interviews: an introduction to qualitative research interviewing. Thousand Oaks: Sage; 1996.

23. Ministério da Saúde. Portaria no 2. 684, de 8 de novembro de 2013. Redefine as regras e os critérios referentes aos incentivos financeiros de investimento para construção de polos e de custeio e no âmbito do Programa Academia da Saúde e os critérios de similaridade entre Programas em Desenvolvimento no Distrito Federal ou no Município e o Programa Academia da Saúde. Diário Oficial da União 2013; 14 nov.

24. U.S. Department of Health and Human Services. Manual para avaliação de programas de atividade física. Atlanta: Centers for Disease Control and Prevention; 2002.

25. Ribeiro IC, Torres A, Parra DC, Reis R, Hoehner C, Schmid TL, et al. Using logic models as iterative tools for planning and evaluating physical activity promotion programs in Curitiba, Brazil. J Phys Act Health 2010; 7 Suppl 2:S155-62.

26. Leviton LC, Collins C, Laird B, Kratt P. Teaching evaluation using evaluability assessement. Evaluation 1998; 4:389-409.
27. Ministério da Saúde. Manual técnico de implantação do Programa Academia da Saúde sobre infraestrutura dos polos. http://portal saude.saude.gov.br/images/pdf/2015/feverei ro/10/Manual-do- programa-Academia-daSa--de---Vol.\%20II\%20- 20INFRAESTRUTU RA\%20DOS\%20POLOS.pdf (acessado em 03/ Mai/2015).

28. Ministério da Saúde. Ministério da Saúde disponibiliza manual técnico de implantação do Programa Academia da Saúde. http://portal saude.saude.gov.br/index.php/o-ministerio/ principal/leia-mais-o-ministerio/1026-secre taria-svs/vigilancia-de-a-a-z/academia-dasaude-svs/16541-ministerio-da-saude-dispo nibiliza-manual-tecnico-de-implantacao-doprograma-academia-da-saude (acessado em 03/Mai/2015).

29. Traverso-Yépez MA. Dilemas na promoção da saúde no Brasil: reflexões em torno da política nacional. Interface Comun Saúde Educ 2007; 11:223-38.

30. Tremblay MS, Barnes JD, Bonne JC. Impact of the active healthy kids Canada report card: a 10-year analysis. J Phys Act Health 2014; 11 Suppl 1:S3-20.

31. Bezerra LA, Cazarin G, Alves CKA. Modelagem de programas: da teoria à operacionalização. In: Samico I, Felisberto E, Figueiró AC, Frias PG, organizadores. Avaliação em saúde: bases conceituais e operacionais. Rio de Janeiro: MedBook; 2010. p. 65-78.

32. Guarda FRB, Silva RN, Silva SM, Santana PR. A atividade física como ferramenta de apoio às ações da Atenção Primária à Saúde. Rev Bras Ativ Fís Saúde 2014; 19:265-70.

33. Silva DMS, Silva RMV, Guarda FRB, Arruda SGB, Petribu MMV. Projeto Cintura Fina: prevenção e controle da obesidade e demais doenças crônicas não transmissíveis. Rev Bras Ativ Fís Saúde 2014; 9:785-6.

34. Radley A. Making sense of illness: the social psychology of health and disease. London: Sage; 1994.

35. Alencar DL, Brito ALRO, Lisboa KWSC. Promoção da saúde na estratégia de saúde da família: percepção da equipe de enfermagem do Crato-CE. Rev Bras Promoç Saúde (Impr.) 2012; 25:420-5.

36. Trevisan M. Evaluability assessment from 1986 to 2006. Am J Eval 2007; 28:290-303.

38. Oliveira LGD, Natal S, Camacho LAB. O programa de controle da tuberculose em unidades prisionais de dois estados brasileiros. Cad Saúde Colet (Rio J.) 2012; 20:250-7. 


\section{Abstract}

Implementation of the Health Gym Program is one of the key strategies in the Brazilian National Health Promotion Policy, but thus far no evaluability studies have been performed on this intervention in Recife, Pernambuco State, one of the first cities in the country to implement this intervention. This study aimed to measure the evaluability of the Health Gym Program in the city of Recife, based on the description of the intervention and the elaboration of its log frame model and theoretical models for evaluation, in addition to identifying evaluative questions. A qualitative approach was used, based on a seven-element system, by document research, interviews, and discussion with the program's managers and staff, as well as the condensation of meanings technique for analysis of the documents and interviews. The analysis showed that the program has a wide range of objectives, principles, and guidelines, and that part of the management ignores such elements. After modeling the program, the study showed the methodological and operational feasibility for the development of an evaluative survey that was discussed and negotiated with managers and workers.

Health Evaluation; Health Policy; Primary Health Care; Health Services Evaluation

\section{Resumen}

La implantación del Programa Academia de la Salud es una de las estrategias para la implementación de la Política Nacional de Promoción de la Salud en Brasil, no obstante, hasta el momento, no se realizaron estudios de disponibilidad sobre esta intervención en Recife, Pernambuco, que se configura como uno de los primeros entes federativos en implantar esta intervención. Este estudio buscó medir la disponibilidad del Programa Academia de la Salud en el municipio de Recife, considerando la descripción de la intervención, la elaboración de su modelo lógico y modelo teórico de evaluación, además de identificar preguntas evaluativas. Se utilizó un enfoque cualitativo, basado en el sistema de siete elementos, por investigación documental, entrevistas y discusión con gestores y técnicos del programa, además de la técnica de condensación de significados para el análisis de los documentos $y$ entrevistas. Se verificó que el programa presenta una extensa gama de objetivos, principios y directrices, y que parte de los gestores desconoce tales elementos. Tras el modelización del programa, se observó la viabilidad metodológica y operacional para el desarrollo de una investigación evaluativa que fue discutida y pactada con los gestores $y$ trabajadores.

Evaluación en Salud; Politica de Salud; Atención Primaria de Salud; Evaluación de Servicios de Salud
Recebido em 26/Set/2015

Versão final reapresentada em 06/Abr/2016 Aprovado em 13/Jun/2016 